\title{
Multiscale Simulations of the Antimicrobial Peptide Maculatin 1.1: Water Permeation through Disordered Aggregates
}

\author{
Daniel L. Parton, ${ }^{\dagger}\|\|$ Elena V. Akhmatskaya, ${ }^{\ddagger}{ }^{\ddagger}$ and Mark S. P. Sansom ${ }^{* \dagger}$ \\ ${ }^{\dagger}$ Department of Biochemistry, University of Oxford, South Parks Road, Oxford OX1 3QU, United Kingdom \\ ${ }^{\ddagger}$ Basque Center for Applied Mathematics (BCAM), Building 500, Bizkaia Technology Park, E-48160 Derio, Spain \\ ${ }^{\S}$ IKERBASQUE, Basque Foundation for Science, E-48011 Bilbao, Spain \\ Supporting Information
}

ABSTRACT: The antimicrobial peptide maculatin 1.1 (M1.1) is an amphipathic $\alpha$-helix that permeabilizes lipid bilayers. In coarse-grained molecular dynamics (CG MD) simulations, M1.1 has previously been shown to form membrane-spanning aggregates in DPPC bilayers. In this study, a simple multiscale methodology has been applied to allow sampling of important regions of the free energy surface at higher resolution. Thus, by back-converting the CG configurations to atomistic representations, it is shown that
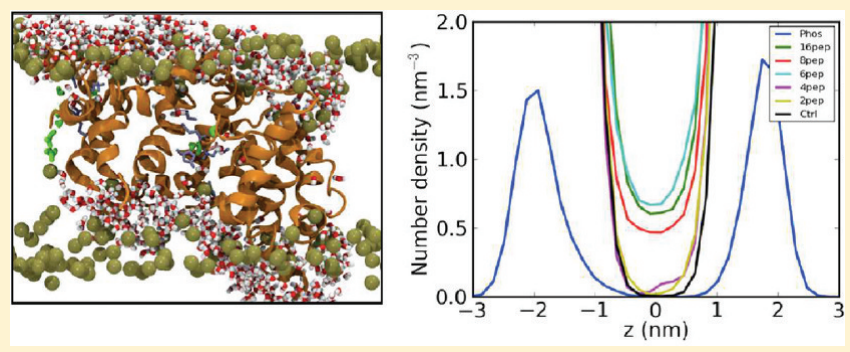
water is able to permeate through the M1.1 aggregates. Investigation of aggregate stoichiometry shows that at least six peptides are required for water permeation. The aggregates are dynamically disordered structures, and water flux occurs through irregular, fluctuating channels. The results are discussed in relation to experimental data and other simulations of antimicrobial peptides.

\section{INTRODUCTION}

Antimicrobial peptides (AMPs) form an important element of the innate immune system of many organisms. Most AMPs are thought to exert their antibacterial effect through permeabilization of the bacterial cell membrane, rather than by binding to a chiral receptor target. This may account for the low level of resistance to AMPs developed by bacteria. ${ }^{1}$ For this reason, there has been interest in the development of both natural and synthetic AMPs as therapeutic agents.

A large group of AMPs form cationic, amphipathic $\alpha$-helices, including the cecropins, ${ }^{2}$ magainins, ${ }^{3}$ and maculatin. ${ }^{4}$ Various models have been proposed to explain the mechanism through which these peptides cause membrane permeabilization in bacteria. Of these, the two main models are the pore-forming and the carpet mechanisms. ${ }^{5}$ The pore-forming mechanism describes the insertion and assembly of peptides to form transmembrane channels, across which water and other cell contents can flow, disrupting the cell. Within this general mechanism, two models further define the nature of the channel. In the barrel-stave model, a transmembrane pore is formed without local disruption of the bilayer structure. In the toroidal pore model, the peptides induce reorganization of nearby lipids so that both peptides and lipid headgroups line the pore lumen. The carpet model suggests that the peptides insert at the membrane surface, parallel to the bilayer, leading to bilayer thinning, and, at high enough peptide concentration, membrane lysis.

The Australian green-eyed tree frog (Litoria genimaculata) secretes an AMP, maculatin 1.1 (M1.1), from its dorsal skin glands, which has antibacterial activity against a wide range of bacteria. ${ }^{5}$ The peptide has the sequence, $\mathrm{GLFGV}^{5} \mathrm{LAKVA}^{10} \mathrm{AHVVP}{ }^{15} \mathrm{AIAEH}{ }^{20} \mathrm{~F}-\mathrm{NH}_{2}$, and its structure, as solved by NMR in both trifluoroethanol/water mixture and dodecylphosphocholine micelle environments, ${ }^{6}$ is an amphipathic $\alpha$-helix of length $\sim 30 \AA$ and with a kink around the central proline. This kink is suggested to be important to the mechanism of permeabilization, as the P15A mutant has much reduced activity. ${ }^{6}$ This may indicate that the kink increases the ability of the peptide to insert into the membrane, ${ }^{7}$ or it may help to provide an optimal amphipathic surface. ${ }^{8}$

A variety of experimental biophysical studies have been conducted to investigate the membrane-lytic properties of the peptide. A consensus on a single mechanism has not been reached, and studies have indicated that multiple modes of action may be available to the peptide, modulated the composition of the membrane. Solid-state NMR spectroscopy studies with model dimyristoylphosphatidylcholine (DMPC) membranes indicated an interfacial location for M1.1. ${ }^{7,9}$ In mixed DMPC/dimyristoylphosphatidylglycerol (DMPG) bilayers, there was a much stronger interaction with the membrane, which appeared to be a result of a dual mechanism: oligomerization of the peptide, possibly as a pore structure, as well as loss of lipid from the membrane. ${ }^{10}$ This would imply

Special Issue: Macromolecular Systems Understood through Multiscale and Enhanced Sampling Techniques

Received: December 22, 2011

Revised: February 19, 2012 
that modes of action corresponding to both the pore-forming and carpet models might occur together. A carpet-type mechanism has been suggested on the basis of ${ }^{31} \mathrm{P}$ NMR studies with live bacteria ${ }^{11}$ and electron microscopy studies. ${ }^{12}$

Other experiments have supported a pore-forming mechanism. Using confocal fluorescence microscopy, M1.1 was shown to cause differential leakage of fluorescent marker compounds from within giant unilamellar vesicles composed of palmitoyloleylphosphatidylcholine (POPC) or POPC/palmitoyloleylphosphatidylglycerol (POPG). ${ }^{8}$ Out of the two markers, the smaller was found to be able to escape from the vesicle, while the larger could not. Large-scale disruption of the vesicle structure was not observed, so a pore-forming mechanism was proposed as the likeliest mechanism. A study using Fourier transform infrared spectroscopy indicated stronger insertion of the peptide into DMPG than DMPC membranes, ${ }^{13}$ with an orientation of the helix axis of $\sim 35^{\circ}$ to the membrane normal, suggestive of a transmembrane pore structure. Other studies highlight how peptide concentration and membrane composition can modify the behavior of the peptide. $^{10,14}$

Molecular dynamics (MD) simulations have enabled development of more detailed models of how AMPs interact with membranes (for a recent review see ref 15). For example, simulations of magainin- $\mathrm{H} 2^{16}$ and melittin ${ }^{17,18}$ have indicated that these peptides are able to form water-permeable pores. However, the time and length scales accessible with all-atom MD simulations are often below those required to observe important processes in biological systems; ${ }^{19}$ for example, the diffusion of peptides within a membrane may be too slow to allow formation of the most stable configuration of peptides.

Coarse-grained (CG) models allow access to much longer time and length scales. One of the first CG models for the simulation of biological membranes was developed in Klein's group $^{20}$ it has been used in simulations of a synthetic arylamide polymer, designed to have similar properties to natural antimicrobial peptides. ${ }^{21}$ A CG force field for lipids, originally developed by Marrink and colleagues, ${ }^{22}$ has been extended by various groups for use with proteins. The same group's MARTINI force field ${ }^{23,24}$ has been used in simulations of magainin, ${ }^{24-26}$ melittin, ${ }^{17}$ and alamethicin. ${ }^{27}$ A similar force field was used in simulations of synthetic peptides by Khalfa et $\mathrm{al}^{28}$ The model used in the current study was also derived from Marrink's original lipid force field. ${ }^{22}$ It was extended for use with proteins by Bond et al. ${ }^{29}$ In all of these models, a single particle is used to represent $\sim 4$ non-hydrogen atoms, with suitable parameters to encapsulate van der Waals, charge, and hydrogen-bonding properties. The current CG model has been used in a wide range of simulations of proteins and membranes. ${ }^{29-37}$

To investigate the mechanism of membrane permeabilization by M1.1 in more detail, Bond et al. performed CG MD simulations of the peptide at various concentrations, and in both bilayer and vesicle environments. ${ }^{31}$ They found that at a low peptide/lipid ratio, M1.1 adopts a kinked, membraneinterfacial location in a phospholipid bilayer, consistent with experiment. $^{7,9}$ At higher peptide/lipid ratios, disordered membrane-spanning aggregates were formed. A simulation with a POPC vesicle revealed that the peptide is capable of inducing membrane curvature and disruption.

While water-flow was not observed and well-defined pores were not evident in the clusters formed by M1.1, the coarsegrained level of representation is likely to have precluded observation of such events. More specifically, such an effect may have been due to the granularity of the CG model (the water particles, equivalent to 4 water molecules, may have been simply too large to flow through membrane pores) and the lack of a water dipole. The latter factor has been shown to be important in the process of pore-formation by AMPs in MD simulations. ${ }^{38}$ The limitations of CG-only simulation approaches for the study of pore formation in membranes have recently been discussed in detail, in relation to the MARTINI force field. ${ }^{39}$

An alternative to CG-only simulations is to adopt a multiscale approach. ${ }^{40}$ As was previously noted by Bond et al., ${ }^{31}$ conversion of a peptide cluster from a CG to an atomistic representation may allow water flow to be investigated in more detail. The results of simulations based on this simple multiscale methodology ${ }^{41}$ are reported here and demonstrate that the disordered peptide clusters are permeable to water. Further simulations help to determine the minimum number of peptides required for water permeation, and the permeation mechanism is discussed in relation to other experimental and computational studies of AMPs.

\section{METHODS}

CG Simulations. The structure of M1.1 used in these simulations was determined by solution-state NMR in a trifluoroethanol/water mixture. ${ }^{6}$ For the CG simulations, the structure was converted to a CG representation, as described in Bond et al. ${ }^{42}$ This CG structure comprised a chain of backbone particles with attached side-chain particles. Harmonic distance restraints (with equilibrium length $0.6 \mathrm{~nm}$ and force constant $1000 \mathrm{~kJ} \mathrm{~mol}^{-1} \mathrm{~nm}^{-2}$ ) were used to restrain the $i$ and $i+4$ backbone particles, mimicking the hydrogen-bonds formed in $\alpha$-helices. ${ }^{43}$ The $i$ to $i+4$ restraint was omitted between Pro- 15 and Ala-11, to model the lack of a hydrogen-bond donor in proline and the consequent greater flexibility of the helix in this region. Histidine side chains were uncharged, and the peptide thus contained a net charge of +1 .

The CG systems were created with a pre-equilibrated, 256lipid, dipalmitoylphosphatidylcholine (DPPC) bilayer, with peptides positioned randomly on each side of and at least 2 $\mathrm{nm}$ from the bilayer. The peptides were added with equal numbers on either side of the bilayer, except for the simulation with three peptides, in which one peptide was added on one side and two on the other. The system was solvated with CG water, and sufficient chloride ions were added to neutralize the net positive charge of the peptides. Three systems were also set up with an excess concentration of $\mathrm{NaCl}$, which was added in solution via replacement of randomly chosen water particles. Prior to production simulation, all CG systems were energyminimized using $<500$ steps of the steepest-descent method, then equilibrated for 20 ns with positional restraints on the peptides and bilayer. A $9 \times 9 \times 26 \mathrm{~nm}$ box size was used for all CG simulations.

CG parameters were taken from Marrink et al. ${ }^{22}$ for lipids, water, and ions and from Bond et al. ${ }^{29}$ for amino acids. CG simulations were run with Lennard-Jones interactions shifted to zero between 0.9 and $1.2 \mathrm{~nm}$, and electrostatics shifted to zero between 0 and $1.2 \mathrm{~nm}$, with a relative dielectric constant of 20 . The nonbonded neighbor list was updated every 10 steps. Simulations were performed at constant temperature, pressure, and number of particles. Temperatures of peptide, lipid and solvent were coupled separately using the Berendsen algorithm at $323 \mathrm{~K}$, with a coupling constant $\tau_{\mathrm{T}}=1 \mathrm{ps}$. The system 


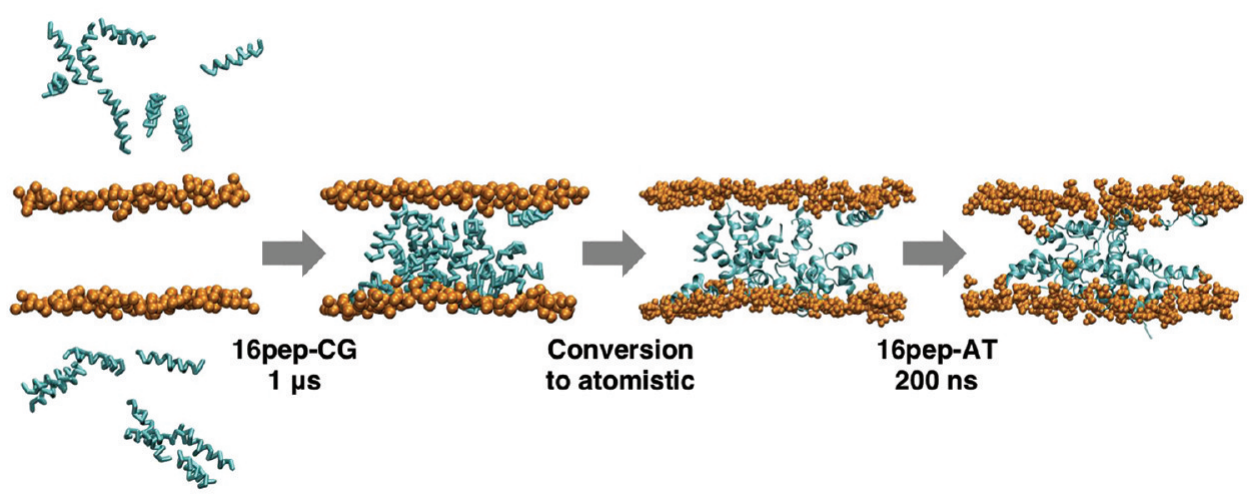

Figure 1. Schematic showing the multiscale protocol used to simulate membrane insertion and permeabilization by M1.1. The leftmost snapshot shows one of the 16pep systems at $t=0 \mathrm{~ns}$. Following a $1000 \mathrm{~ns}$ CG simulation, a representative snapshot (at $920 \mathrm{~ns}$, shown in the second snapshot from left) is converted to an atomistic representation (third snapshot). Atomistic simulations allow water flow to be studied in detail. The final configuration (200 ns) of the same 16pep system is shown in the rightmost snapshot. For clarity, only the peptides (cyan) and lipid phosphate particles (orange) are shown.

Table 1. Water Permeation through the Peptide Clusters ${ }^{a}$

\begin{tabular}{|c|c|c|c|c|}
\hline $\begin{array}{l}\text { simulation }(\times \text { number } \\
\text { of simulations })\end{array}$ & $\begin{array}{l}\text { mean number of } \\
\text { waters in bilayer }\end{array}$ & $\begin{array}{l}\text { mean number of bidirectional water } \\
\text { crossings per nanosecond }\end{array}$ & $\begin{array}{l}\text { diffusional permeability for a single } \\
\text { channel, } p_{\mathrm{d}}\left(\times 10^{-14} \mathrm{~cm}^{3} \mathrm{~s}^{-1}\right)\end{array}$ & $\begin{array}{l}\text { diffusional permeability } \\
\text { coefficient, } P_{\mathrm{d}}\left(\times 10^{-2} \mathrm{~cm} \mathrm{~s}^{-1}\right)\end{array}$ \\
\hline 2pep-AT $(\times 3)$ & $2 \pm 2$ & $0.51 \pm 0.38$ & $0.77 \pm 0.57$ & \\
\hline 4pep-AT $(\times 3)$ & $3 \pm 3$ & $0.42 \pm 0.25$ & $0.63 \pm 0.38$ & \\
\hline 6pep-AT $(\times 3)$ & $27 \pm 12$ & $3.0 \pm 2.0$ & $4.6 \pm 3.0$ & \\
\hline 8pep-AT $(\times 3)$ & $20 \pm 6$ & $2.7 \pm 0.7$ & $4.0 \pm 1.0$ & \\
\hline 16pep-AT $(\times 5)$ & $26 \pm 6$ & $3.4 \pm 1.5$ & & $7.9 \pm 3.6$ \\
\hline $\mathrm{DPPC}(\times 3)$ & $1 \pm 2$ & $0.51 \pm 0.14^{b}$ & & $1.2 \pm 0.3$ \\
\hline
\end{tabular}

${ }^{a_{\text {The }}}$ mean number of waters present in the bilayer was calculated by counting the number of waters between two planes, drawn parallel to the bilayer, centered at the bilayer center (as defined by the average position of the lipid tail terminal carbons), and spaced $1 \mathrm{~nm}$ apart. The calculation was conducted separately for each simulation, then averaged. Water crossings were analyzed by first defining a cylinder of $2 \mathrm{~nm}$ length, oriented with the bilayer normal, centered laterally (in the bilayer plane) at the peptide cluster center and longitudinally at the bilayer center, and of radius roughly equivalent to the lateral radius of the peptide cluster (ranging from 1.7 to $4.5 \mathrm{~nm}$ ). The cylinder is thus a crude representation of a pore through the bilayer at the location of the peptide cluster. A crossing was counted if a water molecule entered one half of the cylinder and exited from the other. The average number of crossings per nanosecond was calculated separately for each simulation, and the resultant values were then averaged. These values and standard deviations were also used to calculate $p_{\mathrm{d}}$ and $P_{\mathrm{d}}$. The single-channel diffusional permeability, $p_{\mathrm{d}}$, was calculated using the relationship $p_{\mathrm{d}}=q_{0} v_{\mathrm{w}}{ }^{62}$ where $q_{0}$ is the number of unidirectional water-crossings per unit time, and $v_{\mathrm{w}}$ is the volume of a single water molecule. The diffusional permeability coefficient for a given area of membrane, $P_{\mathrm{d}}$, can be calculated experimentally by measuring the flux of a tracer molecule across a membrane. Here, the permeability coefficient is calculated using the expression $P_{\mathrm{d}}=q_{0} v_{\mathrm{w}} / A$, where $A$ is the cross-sectional area of the pore, as defined by the cylinder used for the water crossing analysis. A derivation of this expression can be found in the Supporting Information. For all analyses, the first $15 \mathrm{~ns}$ of each simulation was ignored. ${ }^{b}$ For comparison with the 16 pep-AT simulations, the same cylinder radius of $4.5 \mathrm{~nm}$ was used for the analysis of the pure DPPC bilayer control simulations. For comparison with the 2pep-AT simulations, the analysis of the control simulations was repeated with a cylinder radius of $1.7 \mathrm{~nm}$, resulting in a mean of $1 \pm 1$ crossings.

pressure was coupled using the Berendsen algorithm at 1 bar, with a coupling constant $\tau_{\mathrm{P}}=1 \mathrm{ps}$ and a compressibility of $5 \times$ $10^{-6} \mathrm{bar}^{-1}$. The time step for integration was $40 \mathrm{fs}$. The times reported in this work are taken directly from the simulations, rather than scaling by a factor of 4 as is sometimes employed in CG simulations.

Atomistic Simulations. Peptides and lipids were converted from CG to atomistic representations using a fragment-based approach, as described by Stansfeld et al. ${ }^{32}$ The atomistic systems were resolvated with SPC water (after reducing the box $z$ dimension to $11 \mathrm{~nm}$ ) and energy-minimized, using $<500$ steps of the steepest-descent method. Chloride and sodium ions were added as necessary to neutralize the net charge of the peptides and to recreate the three systems with excess salt concentration. Two equilibrations using MD were performed, each for 2 ns. In the first, position restraints (with force constant $1000 \mathrm{~kJ} \mathrm{~mol}^{-1} \mathrm{~nm}^{-2}$ in each direction) were placed on all peptide non-hydrogen atoms; in the second, only the $\alpha$ carbons of each residue were restrained, allowing some relaxation of the peptides without disturbing the structure of the cluster as a whole. Production simulations of up to $200 \mathrm{~ns}$ were then performed.

The force field used in the atomistic simulations was the united-atom GROMOS96 force field ${ }^{44}$ with parameters for DPPC from Berger et al. ${ }^{45}$ Lennard-Jones interactions were truncated beyond $1 \mathrm{~nm}$, while electrostatic interactions beyond this point were approximated using the particle mesh Ewald (PME) summation. The nonbonded neighbor list was updated every 10 steps. Simulations were performed at constant temperature, pressure, and number of particles. Temperatures of peptide, lipid, and solvent were coupled separately using the Berendsen algorithm at $323 \mathrm{~K}$, with a coupling constant $\tau_{\mathrm{T}}=$ $0.1 \mathrm{ps}$. The system pressure was coupled using the ParrinelloRahman algorithm at 1 bar, with a coupling constant of $\tau_{\mathrm{P}}=1$ ps and a compressibility of $4.5 \times 10^{-5} \mathrm{bar}^{-1}$. The time step for integration was 2 fs.

Software and Analysis. All simulations were performed using the GROMACS (www.gromacs.org) simulation pack- 


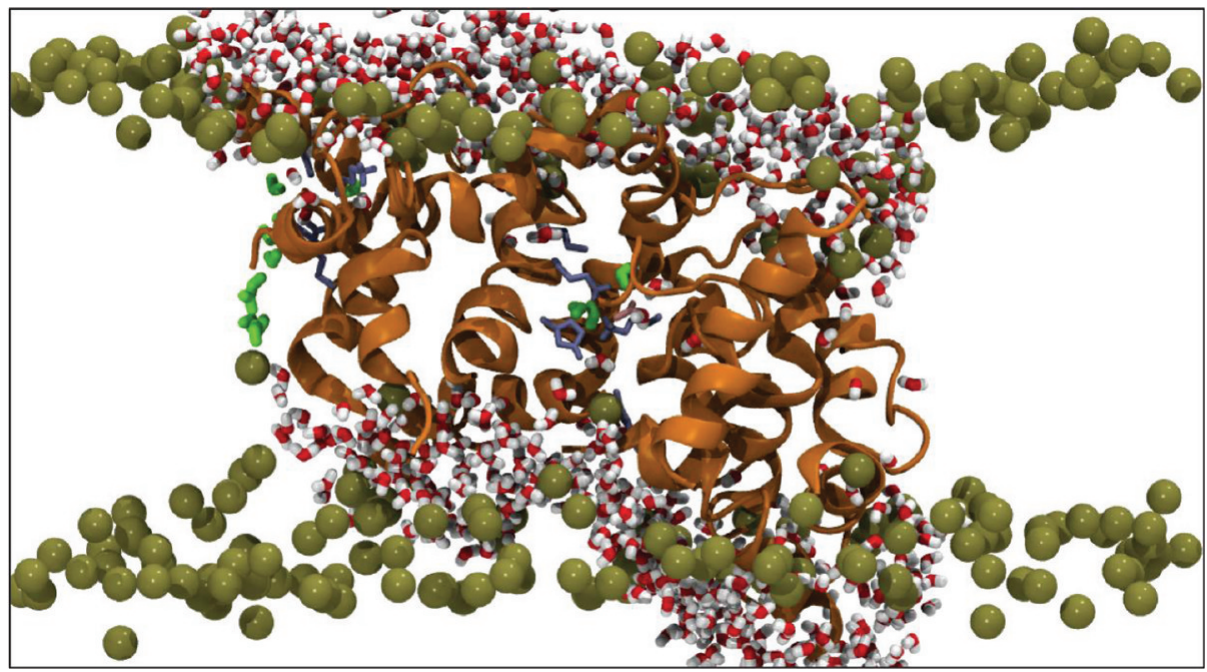

Figure 2. Snapshot of a 16-peptide M1.1 cluster after 24 ns of atomistic simulation. Peptides are shown in orange and DPPC phosphorus atoms in tan. Waters that flow across the cluster during the $30 \mathrm{~ns}$ simulation are shown in green. Nearby lysine and histidine side chains are shown in pale blue; nearby glutamic acid side chains are shown in pink. Other waters near the peptides are shown in red and white. Bulk water is not shown.

age. ${ }^{46-49}$ VMD was used for visualization. ${ }^{50}$ Clustering of the peptide aggregate structures was carried out by fitting the CG trajectories (from the time of complete peptide insertion) to the final configuration of peptides, then using the rmsd-based clustering algorithm g_cluster (part of the GROMACS package) with the single linkage algorithm to populate clusters. The most populated cluster of each simulation was chosen for conversion to atomistic resolution. Secondary structure analysis was performed using the DSSP program. ${ }^{51}$ Water permeation and flux analysis was carried out using the g_count and g_flux software. ${ }^{52}$ Other analysis was carried out using Python with the MDAnalysis library. ${ }^{53}$

\section{RESULTS}

Multiscale Simulations of Peptide/Bilayer Interactions. Five CG systems were set up, each with $16 \mathrm{M} 1.1$ peptides positioned randomly outside a 256 lipid DPPC bilayer. Production simulations of $1 \mu$ s were performed for each system. In each case, the peptides rapidly aggregated in solution and located themselves at the bilayer surface, before inserting as clusters. All 16 peptides eventually became included in a single, membrane-spanning cluster (Figure 1). The clusters formed were irregular, fluctuating structures, with the peptides adopting a wide range of tilt and kink angles. The details of the insertion pathway were as described in a previous study. ${ }^{31}$

For each of the inserted peptide aggregates, a representative configuration was selected using a clustering algorithm and subsequently converted to atomistic resolution (these techniques are described further in the Methods section). Of these five atomistic systems (named 16pep-AT), two were simulated for $200 \mathrm{~ns}$ and the remaining three for $30 \mathrm{~ns}$. In each simulation, water quickly penetrated into sites within the peptide clusters during the first 2 ns equilibration stage. An average of $26 \pm 6$ waters were present in the bilayer region during the simulations, significantly higher than in control simulations of a pure DPPC bilayer, in which an average of one water was present (Table 1 ). Figure 2 shows a representative snapshot. The average density of waters across the bilayer is shown in Figure 3 (green line) and is seen to be much higher in the bilayer center than in the control simulations (black line). The atomistic control system was converted from a CG

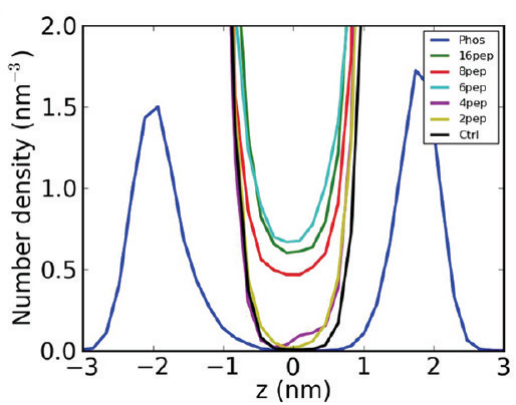

Figure 3. Water density along the $z$ axis (bilayer normal) during the atomistic simulations, averaged over time and each simulation repeat. The first $15 \mathrm{~ns}$ of each simulation were disregarded. The blue line represents the density of DPPC phosphorus atoms for one of the 16pep-AT simulations. Bilayers were centered in the $z$ coordinate in the trajectories used to calculate the density plots.

simulation in the same way as for the peptide simulations and then simulated three times (each with different starting velocities) for $30 \mathrm{~ns}$.

Peptides were able to move within the clusters individually but remained aggregated. The overall features of the clusters were consistent throughout the simulations: irregular structures with the peptides distributed approximately evenly across the bilayer normal. The $200 \mathrm{~ns}$ simulations showed that although peptides were able to move individually within the clusters, these overall features remained conserved over longer time scales (see snapshots in Figure 4a). For each peptide, at least one terminus tended to be anchored at the membrane interface. The degree of helicity of individual peptides within a cluster was varied but did not change to a large extent over the course of the simulations (mean helicity 46\%; Figure 5).

Water Permeation Through Peptide Clusters. Water molecules were able to flow across the clusters through multiple, fluctuating channels, as indicated in Figure 2. The channels were narrow, usually allowing only one water molecule to pass through at a time (although multiple channels would sometimes be available within a peptide cluster). Channels generally remained permeable for the duration of 
a)
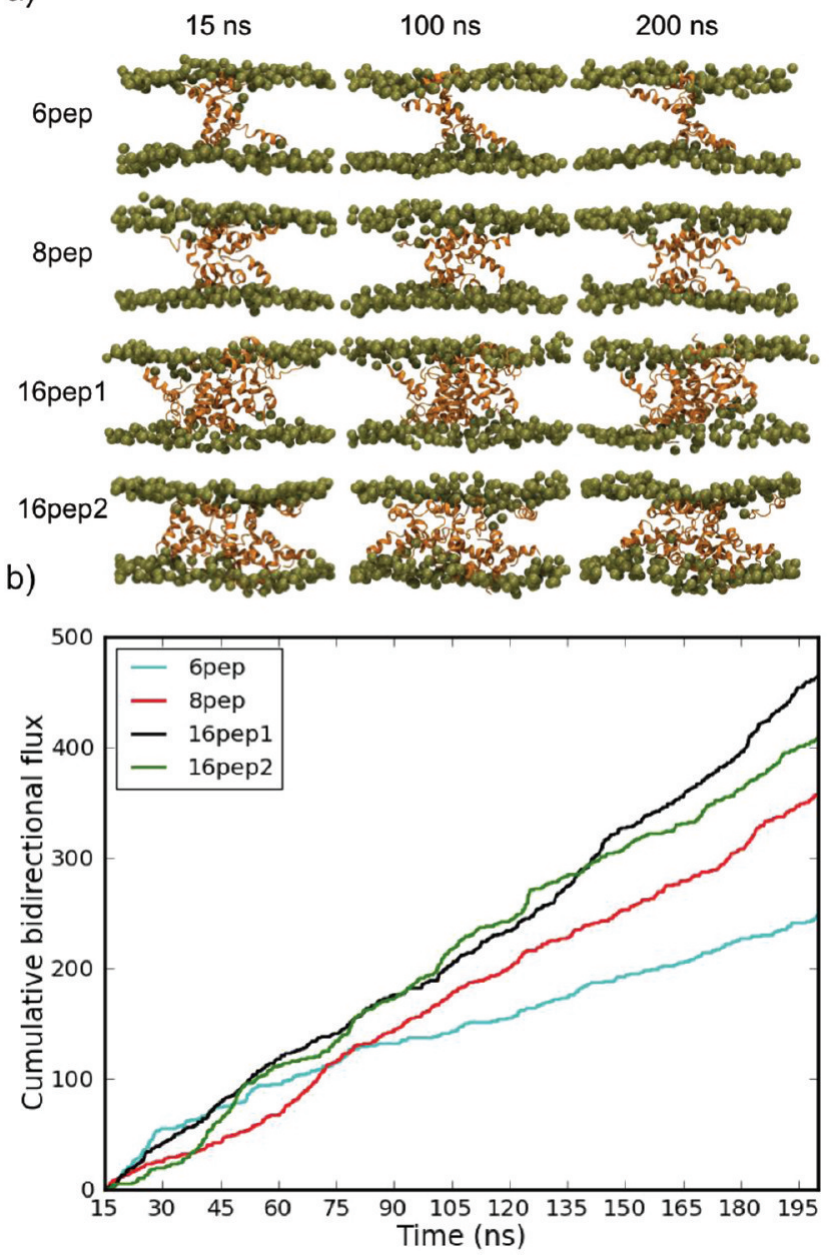

Figure 4. (a) Snapshots of the four $200 \mathrm{~ns}$ atomistic simulations (from the 6pep-AT, 8pep-AT, and 16pep-AT systems), shown at 15, 100, and 200 ns. Peptides are shown in orange and lipid phosphorus atoms in tan. (b) Cumulative water flux over the duration of the $200 \mathrm{~ns}$ simulations, calculated as described in the caption of Table 1 . The first $15 \mathrm{~ns}$ of each simulation is ignored.

the simulations, but the exact path would often fluctuate with the movements of peptides within the cluster.

Two main factors appeared to be important in aiding the passage of water across the membrane. Polar and charged peptide side chains (Lys8, His12, Glu19, and His20) distributed across the membrane core provided a hydrophilic pathway for waters to cross. This is reflected in the higher numbers of water contacts recorded for those residues (Figure 6) and is especially evident for the central Lys8 and His12 when compared to the surrounding residues. Some hydrophobic residues also had relatively high numbers of water contacts, but these were toward the ends of the peptides, which were often anchored at the membrane and thus close to the bulk water.

The other factor aiding the passage of water was the presence of inserted lipid headgroups, which were noticeable in channels formed at the edges of peptide clusters. The insertion of lipids into the membrane core is reminiscent of the disordered toroidal pores observed in simulations of magainin- $\mathrm{H} 2^{16}$ and melittin. ${ }^{17,18}$ However, these inserted lipids and their associated channels were rare in the 16pep-AT simulations, and the majority of water flux occurred through channels entirely within the clusters without inserted lipid headgroups present
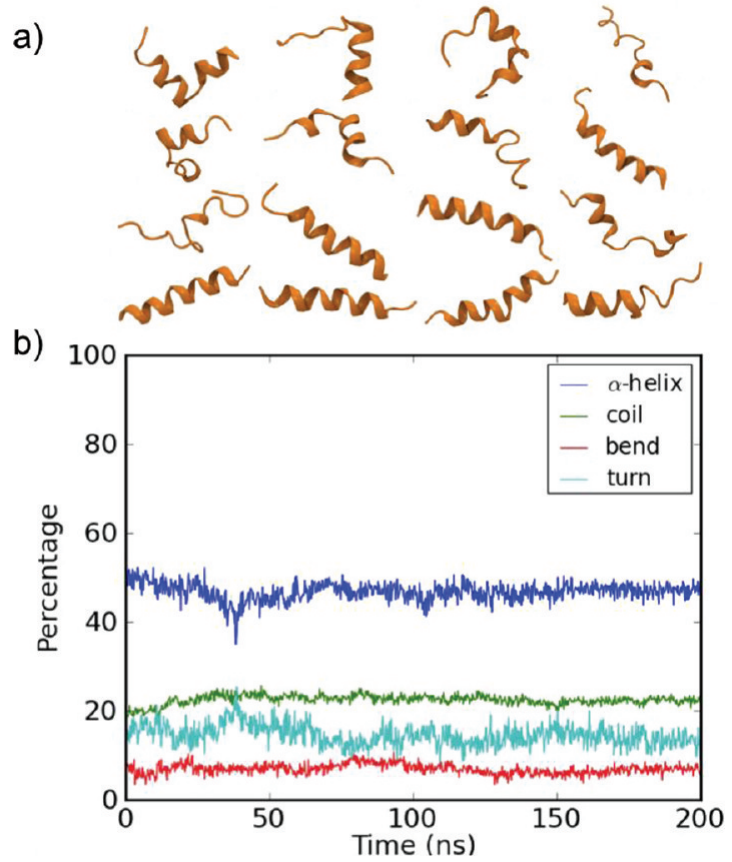

Figure 5. (a) Snapshots of each of the 16 peptides at the end of one of the $200 \mathrm{~ns} 16$ pep-AT simulations, showing the variation in structure. Many peptides are kinked around the central proline. The peptides are shown from the side (i.e., the vertical coordinate of the snapshots is equivalent to the bilayer normal), and they have been rotated around the bilayer normal for clarity. The peptides all make up part of a transmembrane cluster in the simulation but are not shown arranged in a particular order. (b) Secondary structure of all 16 peptides during the same 16pep-AT simulation.

(although more lipid insertions were observed in simulations with 6 peptides, as will be described later in the Results section).

Water flux was quantified by counting the number of bidirectional crossing events during the simulations (Table 1). The diffusional permeability coefficient for the region of membrane containing the inserted cluster was calculated to be $7.9 \times 10^{-2} \mathrm{~cm} \mathrm{~s}^{-1} \pm 3.6$ (Table 1). The value for the control simulations was calculated to be $1.2 \times 10^{-2} \mathrm{~cm} \mathrm{~s}^{-1} \pm 0.3$, which is in agreement with previous atomistic simulations ${ }^{54}$ and experimental estimates. ${ }^{55,56}$ Thus, the peptide cluster appears to increase the local membrane water permeability by a factor of $\sim 6.5$. Considering the evolution of water flux over longer time scales, the rate was approximately constant over the duration of the two $200 \mathrm{~ns}$ simulations, reflecting the consistent structural features of the clusters (Figure $4 \mathrm{~b}$ ).

Minimum Number of Peptides Required for Formation of a Membrane-Spanning Cluster. To investigate the effect of the number of constituent peptides on cluster behavior, CG systems with two, four, six, and eight peptides (named 2pep-CG, 4pep-CG, 6pep-CG, and 8pep-CG respectively; Figure 7) were each simulated three times for $1 \mu \mathrm{s}$.

In the 2pep-CG simulations, the peptides quickly inserted at the bilayer interface, with one positioned in each leaflet. The peptides behaved in a similar fashion to previous simulations of a single M1.1 peptide with a DPPC bilayer, ${ }^{31}$ adopting an orientation parallel to the bilayer plane with only transient periods of further insertion. The peptides were thus unable to contact each other through the bilayer during the simulation, and no aggregation was observed. 
b)

a)
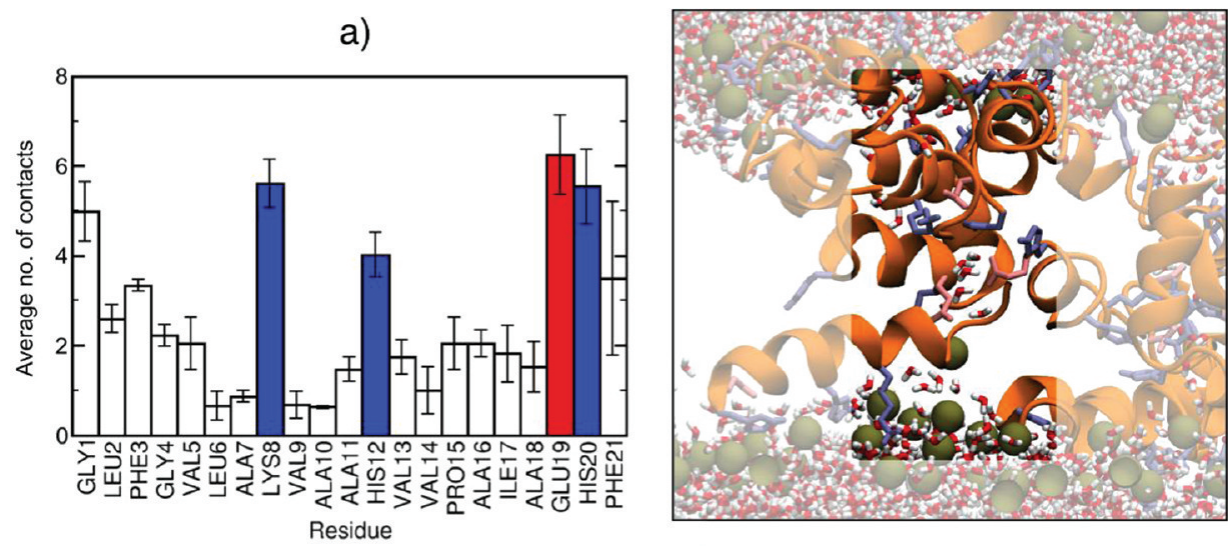

Figure 6. (a) Average number of residue-water contacts per peptide during each of the five 16pep-AT simulations. Contacts were summed over each peptide and simulation (disregarding the first $15 \mathrm{~ns}$ of each) before calculating the average. Contacts with positively charged residues are shown in blue; contacts with negatively charged residues in red; contacts with hydrophobic residues in white. A contact is defined as when any atom of a water molecule is within $0.4 \mathrm{~nm}$ of any atom of an amino acid residue, with a maximum possible count of one per frame for each residue-water pair. (b) Snapshot from one of the 16pep-AT simulations showing waters inserted in the membrane around charged peptide residues. Peptide backbones are shown in orange ribbon format, with the side chains of His-12, Glu-19, and His-20 shown in pale blue and that of Lys-8 in pink. Waters are shown in red and white licorice format, and lipid phosphate atoms as tan spheres.

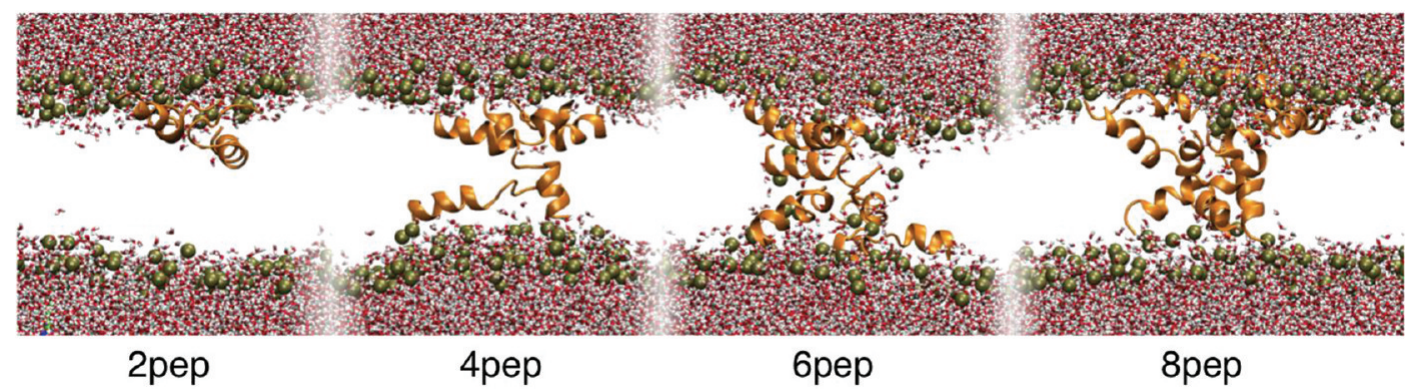

Figure 7. (left to right) Snapshots of clusters formed during the 2pep-AT, 4pep-AT, 6pep-AT, and 8pep-AT simulations, respectively. Peptides are shown in orange, and water molecules in red and white. Lipid phosphorus atoms are shown as tan spheres, but the remainder of the lipid structure is not shown for clarity. In the 6pep-AT snapshot, it can be seen that a number of lipids have rearranged so that their headgroups line the peptide cluster.

The 4pep-CG simulations all resulted in four-peptide clusters, spanning the membrane with two peptides in either leaflet. Clustering was used to select snapshots from each, which were then converted to atomistic resolution (the subsequent simulations being named 4 pep-AT). In one of the 4pep-CG simulations, one pair of peptides first aggregated and inserted into one leaflet of the membrane, while the other pair of peptides did not insert into the other leaflet of the membrane until after 600 ns. This gave the possibility of studying a two-peptide cluster in atomistic detail, so a copy of the first $600 \mathrm{~ns}$ of the trajectory was created and processed to remove the nonmembrane-bound peptides (which were too distant to be influencing the inserted peptides or bilayer), and clustering was used to select three representative snapshots for conversion to atomistic resolution (the subsequent simulations being named 2pep-AT). The 6pep-CG and 8pep-CG simulations also resulted in membrane-spanning clusters, with roughly equal distributions of peptides across the two leaflets. The three 6pep-AT and three 8pep-AT simulations were derived from representative atomistic conversions of each of these systems.

A CG simulation with three peptides was also performed. In this case, two peptides inserted as a cluster in one leaflet, while the other peptide inserted in the opposite leaflet. The single peptide did not reach far enough into the bilayer center to contact with the other peptides, and thus, a membranespanning cluster did not form.

Effect of Cluster Stoichiometry on Water Diffusion. Three atomistic systems were thus available for each of the cluster stoichiometries described above (2pep-AT, 4pep-AT, 6pep-AT, and 8pep-AT). In each case, one system was simulated for $200 \mathrm{~ns}$, and the remaining two were simulated for $30 \mathrm{~ns}$. The same analyses as used for the 16pep-AT simulations were used to determine the water density, number of water molecules present in the bilayer center, and the level of water flux (Figure 3 and Table 1). In the 2pep-AT and 4pepAT simulations, water penetration and flux were similar to the base rate in the control simulations. A large increase was observed for the 6pep-AT simulations in which a number of lipids were inserted into the membrane core surrounding the peptide clusters (Figure 7). The majority of water flow in these simulations occurred through channels formed by these lipid headgroups and the edges of the peptide cluster. However, lipid insertions did not occur in the 8pep-CG (or 8pep-AT) simulations, which gave rise to fairly similar levels of water flux. This finding fits with the results from the 16pep-AT 
simulations, in which lipid insertions could contribute to water flux but were not essential.

Over the course of the $200 \mathrm{~ns}$ simulations, the 6pep-AT system showed a decrease in rate after 30 ns but was subsequently fairly constant, whereas flux in the 8pep-AT system was roughly constant throughout (Figure $4 \mathrm{~b}$ ). This behavior reflected the configurational properties of the peptide clusters. While individual peptides were able to move and sample a range of conformations, the clusters remained as aggregated, membrane-spanning structures, and water channels were irregular and fluctuating.

Rather than calculating permeability coefficients for the smaller peptide clusters (which would be less appropriate for smaller areas of the membrane), they have been treated as single water-channels by evaluating the area-independent diffusional permeability $p_{\mathrm{d}}$ (Table 1$)$. This is directly proportional to the flux (see caption of Table 1), but it is useful to compare it with other simulation and experimental studies. For example, aquaporin-1 has been found to have a $p_{\mathrm{d}}$ of $\sim 6 \times$ $10^{-15} \mathrm{~cm}^{3} \mathrm{~s}^{-1}$. 57,58 The values for the 6- and 8-peptide clusters are almost an order of magnitude higher than this.

Effects of Varying Salt Concentration. It has been shown experimentally that many AMPs display a decreased binding affinity for membranes at high salt concentrations (e.g., for magainin $\left.{ }^{59}\right)$. An atomistic simulation study of magainin bound to a POPC membrane indicated slight but noticeable changes in the positioning of the (unaggregated) peptide at the bilayer/water interface. ${ }^{60}$ We therefore investigated the possible effects of ion concentration on the behavior of maculatin in our simulations. Three sets of simulations were carried out with 16 peptides and a range of salt buffer concentrations (0.25, 0.4, and $0.5 \mathrm{M}$ ). These were carried out according to the same protocol as for the previous 16pep simulations. During the CG simulations, the peptides followed a similar aggregation and insertion pathway to that observed in the previous simulations without excess salt concentration. The overall topologies of the inserted aggregates also appeared to be unchanged by the presence of salt (Figure S1, Supporting Information), although the relatively high degree of fluctuation and disorder in the aggregates may have masked more subtle effects. During the subsequent $30 \mathrm{~ns}$ atomistic simulations, the degree of water permeation and flux through the aggregates was also similar to that observed previously (Table S1, Supporting Information). It therefore appears that salt concentration has a negligible effect on the mechanism of membrane permeabilization observed in these simulations.

\section{DISCUSSION}

The mechanism of membrane permeabilization by maculatin and related peptides has been discussed widely in the literature, with various experimental studies indicating pore-forming or membrane-disrupting models, often showing dependence on conditions such as lipid composition or peptide concentration (see ref 61 for a full review). Although many studies have implied the possibility of pore-formation by the peptide, little direct evidence is available regarding the structure of these membrane-inserted aggregates. This work indicates that M1.1 will form disordered, membrane-spanning clusters, which are permeable to water when containing six or more peptides. Permeation occurs through fluctuating channels, formed either by inserted polar and charged side chains within the peptide clusters or between the cluster edges and inserted lipid headgroups. The inserted peptides adopt a wide range of orientations relative to the membrane. A number of repeat simulations were conducted for each peptide aggregate system, and the extension of a sample of simulations to longer time scales (200 ns) showed consistency of the overall features of the peptide aggregates in terms of structure and water permeability.

This model modifies earlier proposals for the mechanism of antimicrobial peptide pore-formation in which the peptides are assumed to adopt transmembrane orientations around a central pore. However, several recent simulation studies have indicated that the structures may be more disordered than originally suggested, e.g., for magainin-H2, ${ }^{16,24}$ melittin, ${ }^{17,18}$ and alamethicin. ${ }^{18,27}$ Solid-state NMR experiments with alamethicin also reinforced the finding that the inserted peptides take on a large range of configurations in relation to the membrane. ${ }^{27}$

It is also worthwhile to consider the titration state of the two histidines present in M1.1. In the current study, these were modeled as neutral (i.e., unprotonated). It is possible that the protonation state of these residues may change upon insertion within a bilayer. However, this has not been definitively measured experimentally for M1.1 and is difficult to estimate. This feature would therefore present an interesting topic for future study, as it is possible that a change in peptide charge could affect the flow of water across the membrane. Previous simulation work has shown that changes in charge can affect the permeabilization mechanism of melittin. ${ }^{17}$

A range of permeabilization properties has been observed in experimental studies, depending on conditions such as peptide concentration and membrane composition. Our simulations hint at a number of possible explanations. First, the permeability of the peptide clusters in the atomistic simulations was dependent on the number of peptides present. The water channels observed were also irregular and variable. It could also be hypothesized that these channels in fact represent the first step in a membrane rupture mechanism, which would only become apparent at much longer time scales and/or under different conditions. For example, turgor pressure would likely influence the rate of water flow across a peptide cluster in vivo. Another mechanism that may contribute to the range of permeabilization behavior is the membrane distortion and disruption seen in previous simulation work with vesicles. ${ }^{31} \mathrm{An}$ effect on membrane curvature was also observed in a recent CG simulation study of magainin, which showed that the peptide can induce budding of vesicles when added on one side of a membrane or pore-formation when added on both sides. ${ }^{25}$ Overall, it seems likely that multiple modes of action are available to M1.1 (and many other AMPs), which may work together in concert or become more individually dominant depending on the conditions. The current study shows that water flow across a disordered peptide cluster is one possible mechanism.

\section{CONCLUSIONS}

This work, alongside recent studies of alamethicin ${ }^{27}$ and magainin- $\mathrm{H} 2,{ }^{26}$ provides an example of the use of a multiscale simulation methodology to study antimicrobial peptides. The long time-scales attainable with CG molecular dynamics (a total of $24 \mu \mathrm{s}$ were simulated in this work) made feasible the simulation of spontaneous formation of membrane-spanning peptide clusters. Subsequent transformation to an atomistic representation (with a total of $1.28 \mu \mathrm{s}$ of simulation time) allowed for the detailed study of the more subtle properties of the system, especially the interactions of water with the 
peptides and membrane. This methodology adds to the CG simulations in this and the previous work ${ }^{31}$ by showing that the inserted peptide clusters can cause permeabilization of the membrane.

\section{ASSOCIATED CONTENT}

\section{S Supporting Information}

Derivation of the formula used to calculate the permeability coefficient $P_{\mathrm{d}}$, used for the analysis in Table 1; snapshots and analysis of additional 16-peptide simulations conducted with varying salt concentrations. This material is available free of charge via the Internet at http://pubs.acs.org.

\section{AUTHOR INFORMATION}

\section{Corresponding Author}

*Phone: +44 (0)1865 613306. E-mail: mark.sansom@bioch.ox. ac.uk.

\section{Present Address}

"Department of Chemistry, University of Chicago, 5735 South Ellis Avenue, Chicago, Illinois 60637, United States.

\section{Notes}

The authors declare no competing financial interest.

\section{ACKNOWLEDGMENTS}

We would like to thank Dr Phillip Stansfeld for use of his script for conversion from CG to atomistic representations, ${ }^{32}$ and Dr Oliver Beckstein for use of his water counting and flux analysis scripts. ${ }^{52}$ We thank BBSRC, Fujitsu Laboratories of Europe, and the Wellcome Trust for funding.

\section{REFERENCES}

(1) Maloy, W. L.; Kari, U. P. Biopolymers 1995, 37, 105-122.

(2) Steiner, H.; Hultmark, D.; Engstrom, A.; Bennich, H.; Boman, H. G. Nature 1981, 292, 246-248.

(3) Zasloff, M. Proc. Natl. Acad. Sci. U.S.A. 1987, 84, 5449-5453.

(4) Rozek, T.; Waugh, R. J.; Steinborner, S. T.; Bowie, J. H.; Tyler, M. J.; Wallace, J. C. J. Pept. Sci. 1998, 4, 111-115.

(5) Oren, Z.; Shai, Y. Biopolymers 1998, 47, 451-463.

(6) Chia, B. C.; Carver, J. A.; Mulhern, T. D.; Bowie, J. H. Eur. J. Biochem. 2000, 267, 1894-1908.

(7) Marcotte, I.; Wegener, K. L.; Lam, Y.-H.; Chia, B. C. S.; de Planque, M. R. R.; Bowie, J. H.; Auger, M.; Separovic, F. Chem. Phys. Lipids 2003, 122, 107-120.

(8) Ambroggio, E. E.; Separovic, F.; Bowie, J. H.; Fidelio, G. D.; Bagatolli, L. A. Biophys. J. 2005, 89, 1874-1881.

(9) Balla, M.; Bowie, J.; Separovic, F. Eur. Biophys. J. 2004, 33, 109116.

(10) Gehman, J. D.; Luc, F.; Hall, K.; Lee, T.-H.; Boland, M. P.; Pukala, T. L.; Bowie, J. H.; Aguilar, M.-I.; Separovic, F. Biochemistry 2008, 47, 8557-8565.

(11) Chia, B.; Lam, Y.; Dyall-Smith, M.; Separovic, F.; Bowie, J. Lett. Pept. Sci. 2000, 7, 151-156.

(12) Pukala, T. L.; Bowie, J. H.; Maselli, V. M.; Musgrave, I. F.; Tyler, M. J. Nat. Prod. Rep. 2006, 23, 368-393.

(13) Chia, C. S. B.; Torres, J.; Cooper, M. A.; Arkin, I. T.; Bowie, J. H. FEBS Lett. 2002, 512, 47-51.

(14) Mechler, A.; Praporski, S.; Atmuri, K.; Boland, M.; Separovic, F.; Martin, L. L. Biophys. J. 2007, 93, 3907-3916.

(15) Bond, P. J.; Khalid, S. Protein Pept. Lett. 2010, 17, 1313-1327.

(16) Leontiadou, H.; Mark, A. E.; Marrink, S. J. J. Am. Chem. Soc. 2006, 128, 12156-12161.

(17) Sengupta, D.; Leontiadou, H.; Mark, A. E.; Marrink, S. J. Biochim. Biophys. Acta 2008, 1778, 2308-2317.

(18) Mihajlovic, M.; Lazaridis, T. Biochim. Biophys. Acta 2010, 1798, $1485-1493$.
(19) Lindahl, E.; Sansom, M. S. P. Curr. Opin. Struct. Biol 2008, 18, 425-431.

(20) Shelley, J. C.; Shelley, M. Y.; Reeder, R. C.; Bandyopadhyay, S.; Klein, M. L. J. Phys. Chem. B 2001, 105, 4464-4470.

(21) Lopez, C. F.; Nielsen, S. O.; Srinivas, G.; DeGrado, W. F.; Klein, M. L. J. Chem. Theory Comput. 2006, 2, 649-655.

(22) Marrink, S. J.; de Vries, A. H.; Mark, A. E. J. Phys. Chem. B 2004, $108,750-760$.

(23) Marrink, S. J.; Risselada, H. J.; Yefimov, S.; Tieleman, D. P.; deVries, A. H. J. Phys. Chem. B 2007, 111, 7812-7824.

(24) Monticelli, L.; Kandasamy, S. K.; Periole, X.; Larson, R. G.; Tieleman, D. P.; Marrink, S. J. J. Chem. Theory Comput. 2008, 4, 819834.

(25) Woo, H.-J.; Wallqvist, A. J. Phys. Chem. B 2011, 115, 81228129.

(26) Rzepiela, A.; Sengupta, D.; Goga, N.; Marrink, S. J. Faraday Discuss. 2010, 144, 431-443.

(27) Thogersen, L.; Schiott, B.; Vosegaard, T.; Nielsen, N.; Tajkhorshid, E. Biophys. J. 2008, 95, 4337-4347.

(28) Khalfa, A.; Treptow, W.; Maigret, B.; Tarek, M. Chem. Phys. 2009, 358, 161-170.

(29) Bond, P. J.; Wee, C. L.; Sansom, M. S. P. Biochemistry 2008, 47, 11321-11331.

(30) Parton, D. L.; Klingelhoefer, J. W.; Sansom, M. S. P. Biophys. J. 2011, 101, 691-699.

(31) Bond, P. J.; Parton, D. L.; Clark, J. F.; Sansom, M. S. P. Biophys. J. 2008, 95, 3802-3815.

(32) Stansfeld, P. J.; Hopkinson, R.; Ashcroft, F. M.; Sansom, M. S. P. Biochemistry 2009, 48, 10926-10933.

(33) Klingelhoefer, J. W.; Carpenter, T.; Sansom, M. S. P. Biophys. J. 2009, 96, 3519-3528.

(34) Psachoulia, E.; Fowler, P. W.; Bond, P. J.; Sansom, M. S. P. Biochemistry 2008, 47, 10503-10512.

(35) Wee, C. L.; Gavaghan, D.; Sansom, M. S. P. Biophys. J. 2008, 95 , 3816-3826.

(36) Kalli, A. C.; Campbell, I. D.; Sansom, M. S. P. Proc. Natl. Acad. Sci. U.S.A. 2011, 108, 11890-11895.

(37) Kalli, A. C.; Hall, B. A.; Campbell, I. D.; Sansom, M. S. P. Structure 2011, 19, 1477-1484.

(38) Jean-Francois, F.; Elezgaray, J.; Berson, P.; Vacher, P.; Dufourc, E. J. Biophys. J. 2008, 95, 5748-5756.

(39) Bennett, W. F. D.; Tieleman, D. P. J. Chem. Theory Comput. 2011, 7, 2981-2988.

(40) Ayton, G. S.; Voth, G. A. Curr. Opin. Struct. Biol. 2009, 19, 138144.

(41) Stansfeld, P. J.; Sansom, M. S. P. J. Chem. Theory Comput. 2011, 7, 1157-1166.

(42) Bond, P. J.; Holyoake, J.; Ivetac, A.; Khalid, S.; Sansom, M. S. P. J. Struct. Biol. 2007, 157, 593-605.

(43) Bond, P. J.; Sansom, M. S. P. J. Am. Chem. Soc. 2006, 128, 2697-2704.

(44) Scott, W. R. P.; Hunenberger, P. H.; Tironi, I. G.; Mark, A. E.; Billeter, S. R.; Fennen, J.; Torda, A. E.; Huber, T.; Kruger, P.; van Gunsteren, W. F. J. Phys. Chem. A 1999, 103, 3596-3607.

(45) Berger, O.; Edholm, O.; Jähnig, F. Biophys. J. 1997, 72, $2002-$ 2013.

(46) Berendsen, H. J. C.; van der Spoel, D.; van Drunen, R. Comput. Phys. Commun. 1995, 91, 43-56.

(47) Spoel, D. V. D.; Lindahl, E.; Hess, B.; Groenhof, G.; Mark, A. E.; Berendsen, H. J. C. J. Comput. Chem. 2005, 26, 1701-1718.

(48) Lindahl, E.; Hess, B.; van der Spoel, D. J. Mol. Modell. 2001, 7, 306-317.

(49) Hess, B.; Kutzner, C.; van der Spoel, D.; Lindahl, E. J. Chem. Theory Comput. 2008, 4, 435-447.

(50) Humphrey, W.; Dalke, A.; Schulten, K. J. Mol. Graphics 1996, 14 (33-8), 27-28.

(51) Kabsch, W.; Sander, C. Biopolymers 1983, 22, 2577-2637.

(52) Beckstein, O.; Sansom, M. S. P. Phys. Biol. 2004, 1, 42-52. 
(53) Michaud-Agrawal, N.; Denning, E. J.; Woolf, T. B.; Beckstein, O. J. Comput. Chem. 2011, 32, 2319-2327.

(54) Bemporad, D.; Essex, J. W.; Luttmann, C. J. Phys. Chem. B 2004, 108, 4875-4884.

(55) Finkelstein, A. J. Gen. Physiol. 1976, 68, 127-135.

(56) Paula, S.; Volkov, A. G.; Van Hoek, A. N.; Haines, T. H.; Deamer, D. W. Biophys. J. 1996, 70, 339-348.

(57) de Groot, B. L.; Grubmüller, H. Science 2001, 294, 2353-2357.

(58) Smolin, N.; Li, B.; Beck, D. A. C.; Daggett, V. Biophys. J. 2008, 95, 1089-1098.

(59) Matsuzaki, K.; Harada, M.; Funakoshi, S.; Fujii, N.; Miyajima, K. Biochim. Biophys. Acta 1991, 1063, 162-170.

(60) Kandasamy, S. K.; Larson, R. G. Biochim. Biophys. Acta 2006, $1758,1274-1284$.

(61) Fernandez, D. I.; Gehman, J. D.; Separovic, F. Biochim. Biophys. Acta 2009, 1788, 1630-1638.

(62) Zhu, F.; Tajkhorshid, E.; Schulten, K. Biophys. J. 2004, 86, 5057. 